\title{
Enhance Security in MANET using Bacterial Foraging Optimization Algorithm
}

\author{
Preeti Gulia \\ Asst. Professor \\ Maharshi Dayanand University \\ Rohtak
}

\author{
Sumita Sihag \\ M.Tech \\ Maharshi Dayanand University \\ Rohtak
}

\begin{abstract}
MANETs (Mobile Ad-hoc Networks) are one of the fastest emerging networks. MANET is an unstructured network in which nodes are mobile and autonomous. Nodes act as hosts as well as routers. This mobility may leads to insecurity in MANET. BFOA (Bacterial foraging optimization algorithm) is a Bio-inspired Algorithm. This algorithm simulates the behavior of bacteria that can be effectively applied in various fields. A new protocol is proposed here, named BFAODV by applying BFOA technique on AODV. The proposed protocol improves the performance metrics in comparisons to DSDV and AODV protocols. This paper detects and prevents from black hole attack using proposed BFAODV algorithm.
\end{abstract}

\section{Keywords}

MANET, BLACKHOLE, BFOA, BFAODV

\section{INTRODUCTION}

A mobile-ad-hoc network (MANET) is a self- organized, self established wireless network consisting of mobile nodes. Nodes act as host as well as router. Communication between mobile nodes by wireless radio links without the use of any pre-established fixed network infrastructure. Compared to wireless networks with other networks in infrastructure, ad-hoc network doesn't require any access points. This property makes these networks highly flexible and robust and makes MANET useful in alot of different applications such as military applications, urgent business and in rescue operations where the existing communication infrastructure has been destroyed or is unavailable, for example after earthquakes and other disasters [2]. Typical MANET nodes are PDAs, Laptops, cellular phones, Pocket PCs, palmtops and Internet Mobile Phones. These devices are lightweight and battery operated [1].

In this paper a technique BFOA is applied on AODV protocol in MANETs which is a bio-inspired algorithm that considers the ability of solving complex problems by cooperation. The interesting point is that the bacteria do not need any direct communication for the solution of problem, instead the communication between these is not direct, but the communication between individuals through modifying or altering their environment. Several algorithms are based on ant colony and swarm intelligence were introduced in recent years to solve complex problems (e.g. optimization problems, including security and routing etc. Results are based on simulations (NS-2).

The rest of the paper is organized as follows. Section 2, describes related work of various bio inspired algorithms for MANET security. Section 3 describes blackhole attack, Section 4, describes protocols of MANETs. Section 5, describes why use BFOA in MANETs. Section 6 proposes a new BFAODV protocol using BFOA approach on AODV. Section 7, shows the Experimental results in the form of table and graphical presentation of packet delivery ratio, overhead and throughput of protocols. Experimental results are simulated in ns-2 simulation tool; finally, conclusion is discussed in Section 8 with suggestions for future scope.

\section{RELATED WORK}

Ad hoc on Demand Distance Vector Routing Protocol (AODV) is one among the effective Reactive Routing Protocol in MANET. Patil proposed a new protocol Enhanced AODV (E-AODV) which is a modified version of AODV with enhanced packet delivery ratio and minimized end to end delay [5]. Nital Mistry et al., proposed modifications to the AODV protocol and justify the solution which shows significant improvement in packet Delivery Ratio (PDR) of AODV in presence of blackhole attacks, with marginal rise in average end-to-end delay. The solution also maintains the identity of the malicious node as Mali_node, so that in future, it can discard any control messages coming from that node and identified. [6] Aws Kanan et al.,apply the Ant Colony evolutionary optimization technique to the routing problem, Ant colony optimization (ACO) are conducted, taking into account several factors to analyze its adaptive nature. ) only the best ant adds pheromone trails, and (ii) the minimum and maximum values of the pheromone are explicitly limited. [7] Khaleel Ur Rahman Khan et al., an extended version of DSDV (eDSDV) Protocol is proposed for Ad Hoc networks, which overcomes the problem of stale routes and improves the performance of regular DSDV [8]. Ponniyin Selvan et al., proposed Modified on-demand routing algorithm for Mobile Ad- hoc Networks (MANETs), Ant Mesh Network AODV is proposed based on ant colony optimization. The modified routing protocol improves the throughput and decreases the packet loss along with reduction [9] Orhan Dengiz et al.,Smith proposed a particle swarm optimization (PSO) algorithm uses the maximum flow objective to choose optimal locations of the agents during each time step of network operation. The proposed MANET management system is rigorously tested on number of static and dynamic problems. Computational results show that the proposed approach is effective in improving the connectivity of MANETs and predicting movements of user nodes and deploying agents accordingly significantly improves the overall performance of a MANET [10].

\section{BLACKHOLE ATTACK}

The blackhole attack is the most affecting and popular attack among many attacks in MANETs networks and damage the networks to a greater extent. The blackhole attack has properties like, first, the node exploits the mobile ad hoc routing protocol, such as AODV, to advertise itself as having a valid route to a destination node or shows itself as an established node in the network topology and make complete refusal to actual node to participate in the network, and this may cause a denial-of-service. Even though the route is spurious, with the intention of intercepting packets. Second, the attacker consumes the intercepted packets without 
forwarding. There is a more serious form of these attacks when an attacker selectively forwards packets[3][5].

\section{PROTOCOLS OF MANET:}

\subsection{Ad- Hoc on Demand Distance Vector (AODV)}

AODV is a reactive routing protocol which is capable of unicast, multicast and broadcast routing. It is an on demand routing algorithm, meaning that it builds routes between nodes only when source nodes demands. It maintains these routes as long as they are needed by the sources. Additionally, AODV forms trees which connect multicast group members. The trees are composed of the group members and the nodes needed to connect the members. Sequence numbers used by AODV to ensure freshness of routes. It is self starting, loop-free, and scales to large numbers of mobile node [3]. AODV uses the route discovery and route reply process to create and maintain a route on demand. In the route discovery phase for a source node to send information to a destination node, it first checks its own routing table to see if a valid route exists. If a valid route does not exist, a source node broadcasts a global RREQ (Route Request) message that contains the source sequence number, source address, destination sequence number, destination address, broadcast ID, and hop count [4]. The combination of the source address and the broadcast-ID is used to uniquely identify each RREQ message. A node that receives the RREQ message which is usually the nearest node to the source node replies immediately with a routing reply (RREP) if it has a fresh route. Otherwise, it forwards the RREQ message to establish the route to the destination. The sender selects the first node to respond with a RREP (Routing Reply). Every RREP control message received, the source node first checks whether it has an entry for the destination in the route table or not. If there is an entry found, the source node would

\subsection{Destination-Sequenced-Distance Vector (DSDV)}

DSDV is a proactive protocol .Every node in the topology maintains the routing information In a table format that's why also named "table driven" protocol. The routing table holds and maintains a complete set of destination address and the list of intermediate nodes needed to reach each destination [4]. The routing tables are updated whenever the network topology changes (i.e. existing nodes are moved or dropped, or new nodes are introduced). Such changes are announced to every other node. This ensures that each sender knows the route in advance of packet transmissions. Because of advance information about route before packet transmission increases the possibility of attacks.

\section{WHY USE BFOA IN MANETs?}

The Bacterial Foraging Optimization Algorithm is new comer among various bio-inspired algorithms and belongs to the field of Bacteria Optimization Algorithms and Swarm Optimization, and more widely to the fields of Computational Intelligence and Metaheuristics. It is related to other Swarm Intelligence algorithms such as Ant Colony Optimization and Particle Swarm Optimization. It has been used in many research areas like colour images quantization, face recognition, engineering design problems. On analyzing these problems's solution by BFOA, results obtained are better than other bio inspired and conventional approaches. It is computationally effective and faster and solves hard numerical problems. There have been many extensions of the approach that attempt to hybridize the algorithm with other Computational Intelligence algorithms and Metaheuristics such as Genetic Algorithm, Particle Swarm Optimization and Tabu Search etc [12]. So inspired from other problem's solution we are using BFOA in MANETs on AODV protocol in this paper and analyze the results obtained.

\section{PROPOSED WORK}

The technique BFOA (bacterial foraging optimization algorithm) is new comer to the biological techniques. The process, in which a bacterium moves by taking small steps while searching for nutrients, is called chemo taxis and key idea of BFOA is mimicking chemo tactic movement of virtual bacteria in the problem search space, individual bacterium communicate to other by sending signals. It is a global optimization algorithm for various optimization problems. This technique is also inspired by the social foraging behaviour like ant colony and particle swarm optimization. It attracts the researchers due to its efficiency in solving real world optimization problems and gives better results than traditional methods of problems solving. The next section explains that how BFOA can be applied in MANET to detect and prevent from BLACKHOLE attack.

\section{Steps of Bacterial Foraging Algorithm:}

There are following three steps in Bacterial Foraging Algorithm after the search strategies like swimming and tumbling. They are

1. Chemo taxis.

2. Reproduction

3. Elimination and dispersal.

1. Chemotaxis: This process simulates the movement of an E. coli cell through swimming and tumbling via flagella. Biologically an E. coli bacterium can move in two different ways. It can swim for a period of time in the same direction or it may tumble, and alternate between these two modes of operation for the entire lifetime. Suppose hi(j, k, l) represents ith bacterium at jth chemotactic, kth reproductive and lth eliminationdispersal step. C(i) is the size of the step taken in the random direction specified by the tumble (run length unit) [11].

2. Reproduction: In this phase size of population will remain same by the process: each of the healthier bacteria (those yielding higher value of the objective function) sexually split into two bacteria and the least healthy bacteria eventually die, which are then placed in the same location.

3. Elimination and dispersal: impulsive changes in the local environment where a bacterium population lives may occur due to various reasons e.g. a significant rise in temperature may exterminate a group of bacteria that are currently in a region with a high focus of nutrient gradients. Events can take place in such a manner that all the bacteria in a region are killed or a group is dispersed into a new location. To simulate this phenomenon in BFOA some bacteria are liquidated at arbitrary with a very small probability while the new replacements are randomly initialized over the search space [11].

\subsection{Proposed Algorithm}

1. FOR EACH NODE BLACKHOLE $=0$;

2. ReceiveReply (Packet $P)\{$

3. if(BALCKHOLE =1 AND P has an entry in Route Table)\{ 
4. $\quad$ select Dest_Seq_No from routing table

5. if(P.Dest_Seq_No $>$ Dest_Seq_No $)\{$

6. IF (RREP NOT SENT)

7. THEN BLACKHOLE $=1$;

8. ELSE

9. update entry of $\mathrm{P}$ in routing table

10. unicast data packets to the route specified in RREP

11. BLACKHOLE $=0$;

12. \}

13. else \{

14. discard RREP

15. \}

16. $\}$

17. else \{

18. if(P.Dest_Seq_No $>=$ Src_Seq_No \{

19. Make entry of $P$ in routing table

20. \}

21. else \{

22. discard this RREP

23. $\}\}\}$

\section{PERFORMANCEMETRICS}

This paper worked on Packet Delivery Ratio, overhead and throughput as the performance metric to evaluate and analyze the performance of various routing protocol. Table below showing the performance metrics of various routing protocols.

\begin{tabular}{|c|l|l|l|l|}
\hline Protocol & $\begin{array}{c}\text { Gene } \\
\text { rated } \\
\text { packe } \\
\text { ts }\end{array}$ & $\begin{array}{c}\text { Receive } \\
d \\
\text { packets }\end{array}$ & $\begin{array}{l}\text { Packet } \\
\text { deliver } \\
\text { y ratio }\end{array}$ & $\begin{array}{l}\text { Ro } \\
\text { uti } \\
\text { ng } \\
\text { ove } \\
\text { rhe } \\
\text { ad }\end{array}$ \\
\hline Dsdv & 10261 & 9573 & 93.295 & $\begin{array}{l}0.0 \\
884 \\
301\end{array}$ \\
\hline Aodv & 11458 & 11341 & 98.978 & 0.1 \\
& & & 9 & $\begin{array}{l}006 \\
78\end{array}$ \\
\hline Bfaodv & 10002 & 9953 & 99.510 & 0.0 \\
& & & 1 & $\begin{array}{l}901 \\
197\end{array}$ \\
\hline
\end{tabular}

Table 1: Comparisons of Performance Metrics

\section{Packet Delivery Ratio}

The ratio of the number of data packets delivered to the destination. This describes the level of delivered data to the destination. Sum of number of packets receive / sum of number of packet send. The greater value of packet delivery ratio means the better performance of the protocol. The graph showing below the ratio of packet delivered in DSDV, AODV AND BFAODV protocol. Among these three protocols the BFAODV's P.D.R is greater. It means the performance of BFAODV is better.

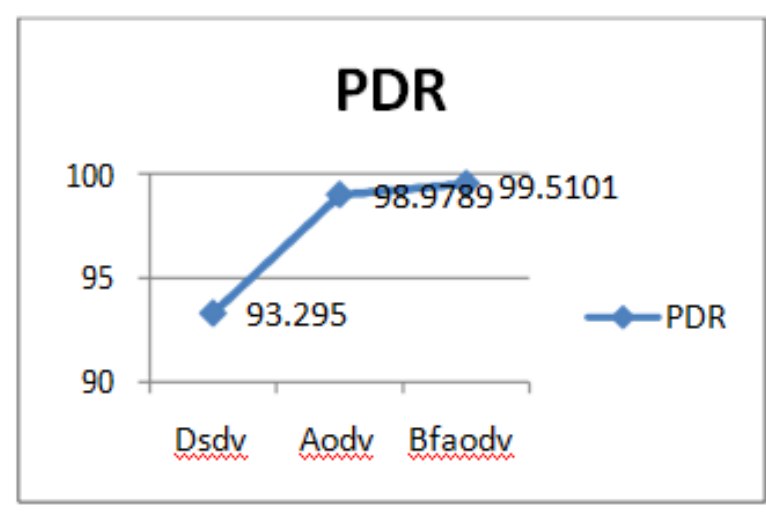

Figure 1: Shows Protocol's PDR

Overhead

Another parameter of performance is overhead. The total number of routing packets transmitted during the simulation experiments. One transmission is count when a packet sent over multiple hops, Throughput of the network is defined as the external measure of effectiveness, efficiency is considered to be the internal measure to achieve a given level of data routing performance, data routing performance may or may not be affected by protocol internal efficiency, If same channel is shared control traffic and data traffic and channel capacity is limited, then excessive control traffic often impacts data routing performance. Slightly greater overhead is shown by BFAODV than overhead of DSDV which is much less than AODV protocol overhead .The minimum overhead is shown by DSDV protocol and maximum is shown by AODV protocol. In this diagram, overhead among three protocols shown below.

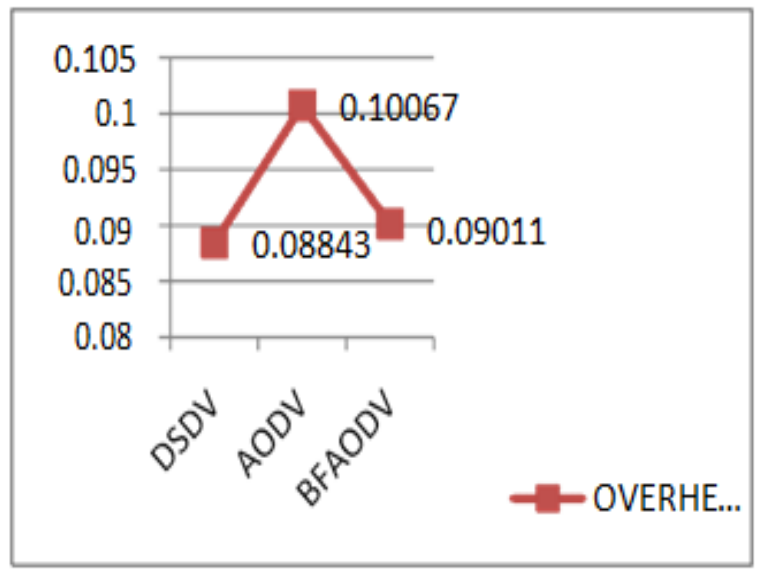

Figure 2: Shows overhead of Protocols

\subsection{Throughput}

It is defined as the total no. of packets over the total simulation time. The throughput comparison of AODV, DSDV and BFAODV shows that which one is better than other two. Maximum the overhead is better the performance of protocol. Among these three BFAODV is better than two. Graph showing below the throughput of BFAODV. 


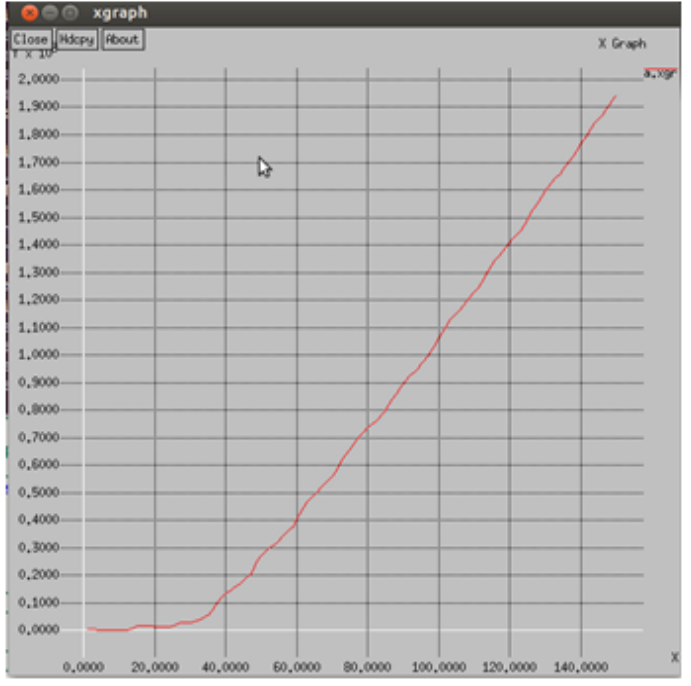

Figure 3: shows throughput of BFAODV

\section{CONCLUSION AND FUTURE SCOPE}

This paper analysis the performance of BFAODV technique in MANETs The nodes movements are like bacteria movement. The technique is applied for detection and prevention from blackhole attack. By applying this technique on MANETs we get better results than Existing MANETs protocols. This improves the performance in terms of packet delivery ratio, overhead and throughput. In future, BFOA will provide to medical data where combination of bacteria foraging and case based reasoning system can be used to diagnose the patient's diseases. This technique may also be apply in areas such as oceanographic astronomical observations and may also apply for solving a broad class of engineering design, software testing and scientific problems and will prove to be a general purpose powerful heuristic method for solving a wider class of such problems. In future artificial intelligence techniques will also propose for security.

\section{REFERENCES}

[1] K.sahadevaiah, "Impact of Security Attacks on a New Security Protocol for Mobile Ad Hoc Networks", Network Protocols and algorithms" ISSN 194335812011, Vol. 3, No. 4

[2] G.S. Mamatha and Dr. S. C. Sharma, "A Highly Secured Approach against Attacks in MANETS", International Journal of Computer Theory and Engineering, Vol. 2, No. 5, October, 20101793-8201.
[3] Neha Ujala Tirkey, 2 Navendu Nitin , 3 Neelesh Agrawal , 4 Arvind Kumar Jaiswal, "Study And Comparison Of Mobile Ad-Hoc Networks Using Ant Colony Optimization" . International Journal of Engineering Research \& Technology (IJERT)Vol. 1 Issue 8, October-2012,ISSN: 2278-0181

[4] Ebrahim Mohammed Louis Dargin, "Routing Protocols Security in Ad Hoc Networks".

[5] Patil V.P, "Efficient AODV Routing Protocol for MANET with enhanced packet delivery ratio and minimized end to end delay", International Journal of Scientific and Research Publications Volume 2, Issue 8, August 2012. ISSN 2250-3153.

[6] Nital Mistry, Devesh C Jinwala, Member, IAENG, Mukesh Zaveri, "Improving AODV Protocol against Blackhole Attacks", proceedings of the international multiconference of engineers and computer scientist 2010, vol II, IMECS, 2010 march 17- 19,2010,hong kong.

[7] Aws Kanan, Taisir Eldos, Mohd Alkahtani, "Mobile Ad Hoc Networks Routing Using Ant Colony Optimization",World of Computer Science and Information Technology Journal (WCSIT) ISSN: 22210741 Vol. 3, No. 6, 105-109, 2010 .

[8] Khaleel Ur Rahman Khan, Rafi U Zaman, "The Performance of the Extended DSDV (eDSDV) MANET Routing Protocol and its Suitability in Integrated InternetMANET", IJSSST, Vol. 10, No. 2 ISSN: 139473 804xOnline, 1473-8031 print.

[9] Ponniyin Selvan and 2S. Senthil Kumar, "An Optimized Ad Hoc on Demand Distance Vector Routing Protocol for Wireless Networks", Journal of Computer Science 8 1177-1183, 2012 ISSN 1549-3636@ 2012 SciencePublications.

[10] Orhan Dengiz a, Abdullah Konak Alice E. Smith c,1 "Connectivity management in mobile ad hoc networks using particle swarm optimization", elsvier Ad Hoc Networks 9 (2011) 1312-1326,2011.

[11] K. Sathish Kumar, T. Jayabarathi,"Power system reconfiguration and loss minimization for an distribution systems", Elsevier, Electrical Power and Energy Systems T. Jayabarathi, Electrical Power and Energy Systems 36 (2012) 13-17.

[12] http://www.cleveralgorithms.com/natureinspired/swarm/bfoa.htm 